\title{
Remote monitoring system for diesel generators at cellular base stations
}

\author{
Syed Shahram Najam ${ }^{1}$, Aamir Zeb Shaikh ${ }^{1, *}$, Shabbar Naqvi ${ }^{2}$, Talat Altaf ${ }^{3}$ \\ ${ }_{1}^{1}$ Department of Electronic Engineering, NED University of Engineering and Technology, Karachi, Pakistan \\ ${ }^{2}$ Department of Computer Systems Engineering, Baluchistan University of Engineering and Technology, Khuzdar, Pakistan \\ ${ }^{3}$ Department of Electronic Engineering, Sir Syed University of Engineering and Technology, Karachi, Pakistan
}

\section{A R T I C L E I N F O}

\section{Article history:}

Received 17 August 2016

Received in revised form

13 July 2017

Accepted 18 July 2017

Keywords:

Fuel Theft

Fuel monitoring system

Current sensor

Lid of generator and micro-switch

\section{Introduction}

In the wake of the energy crisis in Pakistan, base stations of cellular telephony are equipped with a back-up generator to provide uninterrupted connectivity to their subscribers even during long hours of electricity breakdown or load shedding (Harijan et al., 2008). These generators usually run on diesel and if not monitored carefully, the recurring cost of fuel may increase to unacceptable levels. We propose a novel solution to monitor the fuel usage in the generator. With a combination of high precision sensors, it keeps track of the volume of fuel filled, consumed with or without generator operation and generated electricity per liter of fuel. Further, this information can be configured to be sent to predefined persons on their mobile phones through SMS. The system works at extremely low voltage and therefore is safe to work in inflammable environment. The remote monitoring solution for diesel generators can be retrofitted in existing equipment with few changes and will result in substantial savings for the mobile operators. Fuel expenses are a major fraction of the recurring expenditure related to cell site operations. This is further increased by the possibility of fuel theft, mishandling fuel system and low performance of the generator (Aher and Kokate, 2012). Following are some of the scenarios resulting in high fuel expense:

\footnotetext{
* Corresponding Author.

Email Address: aamirzeb@neduet.edu.pk (A. Z. Shaikh) https://doi.org/10.21833/ijaas.2017.08.019

2313-626X/C 2017 The Authors. Published by IASE.

This is an open access article under the CC BY-NC-ND license

(http://creativecommons.org/licenses/by-nc-nd/4.0/)
}

1. The person responsible for the refueling diesel may not refill the correct volume of fuel against the paid amount of money.

2. Someone steals fuel from the tank.

3. There may be any minor leakage in the tank

4. The fuel tank cap may not be secured properly resulting in fuel loss through evaporation.

5. Due to wear and tear in generator and/or not running at rated load, the generator fuel efficiency (kVA generated/liter of fuel) is low.

\section{The proposed system}

Our proposed solution is an electronic system which is equipped with a number of sensors, a logic processor, power module and a GSM module. This system will generate notification in case of fuel wastage due to any of the scenario mentioned above.

1. Whenever, the tank lid is opened and closed, the level of the fuel will be noted at the time of opening of the lid and after the lid is closed. The change in volume will be sent through SMS to the subscriber in terms of liters or percentage of the tank capacity.

2. Whenever the fuel level drops in the tank, whether the generator is off, the difference will be noted and notified to the subscriber via SMS.

3. If the lid is accidently/purposefully left opened, a notification SMS will be sent to the subscriber.

4. When the generator starts, the level of the fuel will be noted. During the running of the generator, voltage and current of the generator will be noted as well. Once the generator is stopped, the amount of fuel used as well as generator performance in 
terms of $\mathrm{kW}$-hour produced per liter will be sent as information SMS to the subscriber.

5. Further feature that are incorporated in the proposed setup includes monitoring of the load (current) on the generator and if the preset load is exceeded a message will be generated informing of the excess load added on the generator.

As shown in the Fig. 1 the system consists of a PIC 16f877a microcontroller i.e. the main controller which is programmed to be the main hub of all calculation, I/O and control of flow of data between various components and a 16x2 LCD display module. The controller unit is also connected to a sensor module consisting of four sensors namely fuel level sensor which will identify the level of fuel in the tank, the energy metering IC (ADE-7755) to check energy consumption of the load, a current sensor (ACS-712) which will be used to monitor the current of the loads being used on the generator and a safety switch which will be installed on the tank lid. The microcontroller module is interfaced with the GSM module as well to transmit performance reports via SMS.

\subsection{Hardware configuration}

In this section, the various and versatile components of the fuel monitoring system will be discussed as it is evident in the previous section and in Fig. 1 that various components are integrated to function and perform in order to obtain the desire output; and every component have to be discussed briefly in order to establish a complete understanding of the working of the Fuel Monitoring System.

The hardware of the fuel monitoring system can be seen in the Fig. 2. A microcontroller is a single integrated circuit outfitted by a microprocessor having streamline qualities of self-sufficiency and relatively lesser in cost (Predko, 1997). Microcontrollers are usually interfaced and embedded in control and monitoring systems as well as processing frameworks.

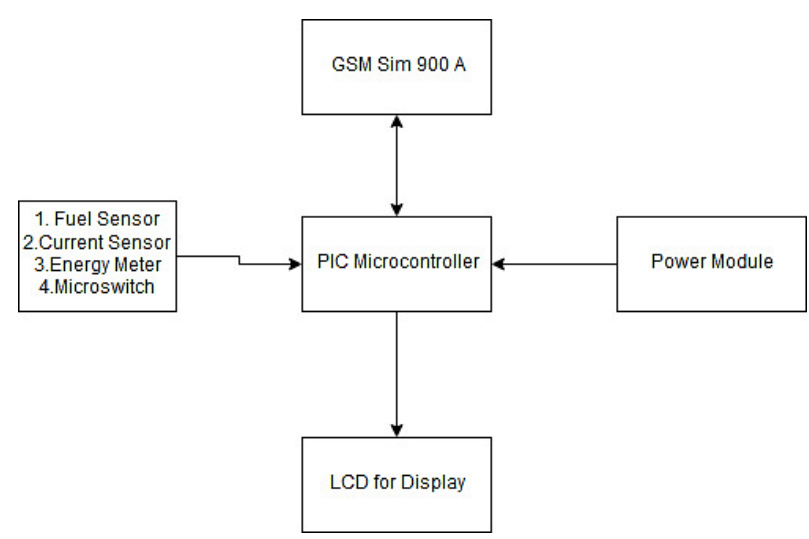

Fig. 1: Block diagram of the fuel monitoring system

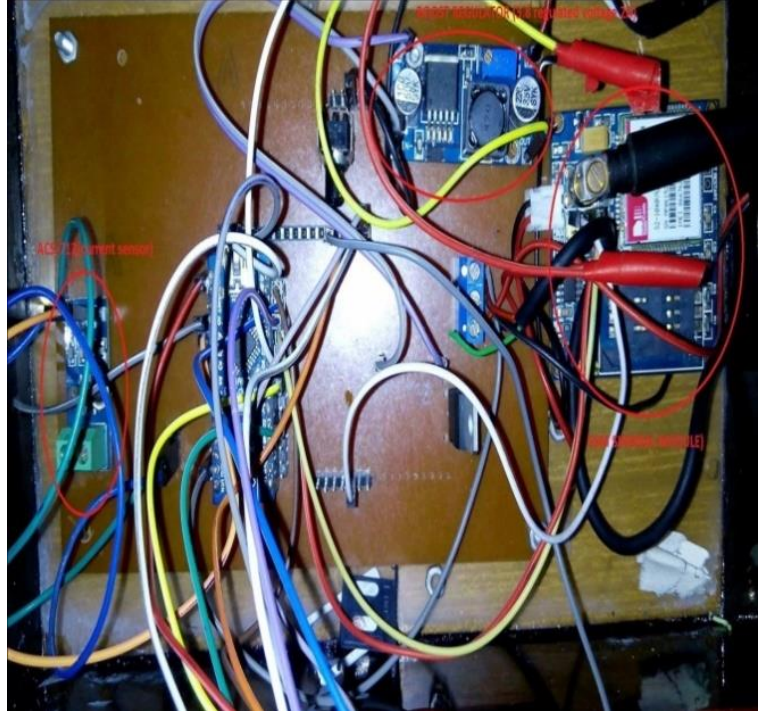

Fig. 2: Proposed fuel monitoring system

\subsubsection{PIC 16f877a}

Microcontroller can be defined as an embedded system with storage and data controlling facility between various peripherals integrated in specific logic i.e. it acts as a co-coordinating body for the circuit (Predko, 1997). With the advancement in the field of micro-electronics and embedded system development, the microcontrollers are not only improved but also a variety of microcontrollers containing embedded processors and memory are available for use of which Microchip's "PIC Series" is one of the widely used (Iovine, 2004).

"PERIPHERAL INTERFACE CONTROLLER" commonly abbreviated in the industry as "PIC" is a series of microcontroller designed by "Microchip" which was initially developed by General Instruments. The reason for using Microchip's PIC series over other series is that firstly it is quite cheap as compared to other microcontrollers being used, secondly it has the optimal processing requirements as well as the proper channels and address lines for interfacing various components like fuel sensor, energy metering IC, Current sensor and GSM module SIM 900 due to having RISC architecture of development (Iovine, 2004). Fig. 3 shows the general hardware configuration of the PIC 16f877a.

\subsection{2. $\mathrm{T} / \mathrm{ll} 141$ (Fuel sensor)}

$\mathrm{T} / \mathrm{ll141}$ is a fuel sensor by FOZMULA. $\mathrm{T} / \mathrm{ll14 \textrm {x }}$ sensors are designed to read the level of fuels like diesel and petrol. The sensor we have used T/ll141 gives an output in the form of voltage (from $0 v-5 v$ ) rather than in the form of capacitance (T/ll140) or in the form of resistance (T/ll142) making it directly interface able with pic 16f877a's analog input (Zvonar et al., 1998).

PIC16F877A having a 10 bit ADC (analog-todigital-convertor), and powered by a 5VDC voltage hence the input from the fuel level sensor mapped from 0 to $5 \mathrm{~V}$ (analog) to 210 i.e. from 0 to 1023. Where 1 unit of digital value from 0 to 1023 will be 
equal to $4.8 \mathrm{mV}$ making it far more accurate as can be seen in the calculation below:

1unit digital $=(5 / 1023) * 1=4.8 \mathrm{mV}$

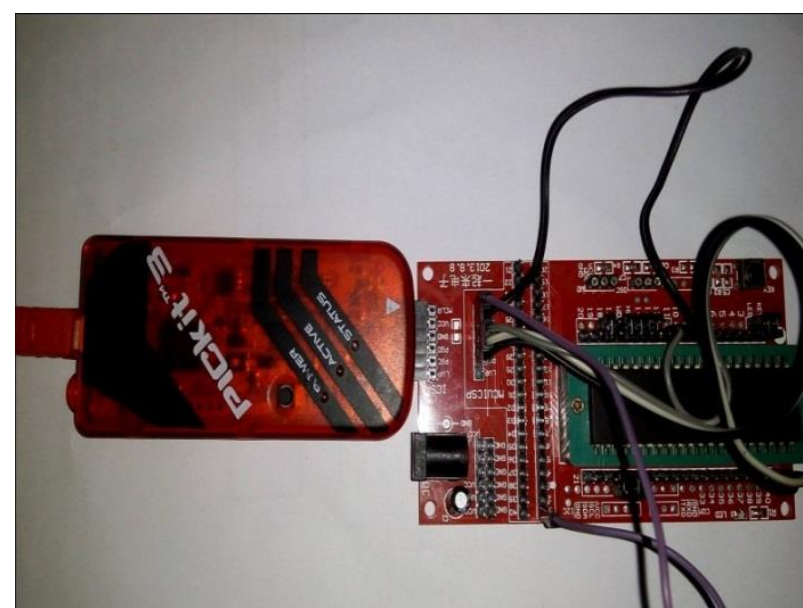

Fig. 3: PIC16F877A development board and programmer (PICKIT 3)

\subsubsection{GSM SIM 900a}

Global System for Mobile Communications (GSM) is one of the most popular cellular communications standard developed by Europe. Presently, this standard is widely used in many countries including Pakistan. This is in addition to the various $3 \mathrm{G}$ and $4 \mathrm{G}$ cellular standards (Garg and Rappaport, 2001). GSM had a global market share of 90\% (Zvonar et al., 1998). This standard is the first digital standard after $1 \mathrm{G}$ (that was primarily an analog FDMA system) for mobile communications. Additionally, several evolving standards make GSM more useful by increasing data rate and adding services through EDGE and GPRS.

The GSM module used in this paper is a device developed by IC station. It is used to transmit/receive short messages using a SIM card issued by a cellular service provider. This module is attached with PIC microcontroller to integrate the GSM capable device to the proposed setup.

The GSM Sim 900a (as shown in Fig. 4) is quite significant in this system as it is the device which sends the data IR has received from the microcontroller (in this case pic 16f877a) and sends it to the user or subscriber regarding the state of the lid or the level of the fuel etc. This SIM900/A V3.8.2 module has a set of TTL level serial interface, a set of RS232 level serial interface, a set of power supply interface and can only support $800 \mathrm{MHz}$ and $1800 \mathrm{MHz}$ only and hence cannot be used in many countries of Europe (Zaghloul, 2014).

\subsubsection{ADE 7755 (Energy metering IC)}

ADE 7755 is a highly accurate IC for measurement of electrical energy. The analog circuitry used is in ADCs while all the signal processing (i.e. multiplication and filtering) is done in digital domain making it quite accurate and stable in extreme conditions i.e. environmental and internal noise generating unwanted frequency to disrupt the output (Biao, 2007).

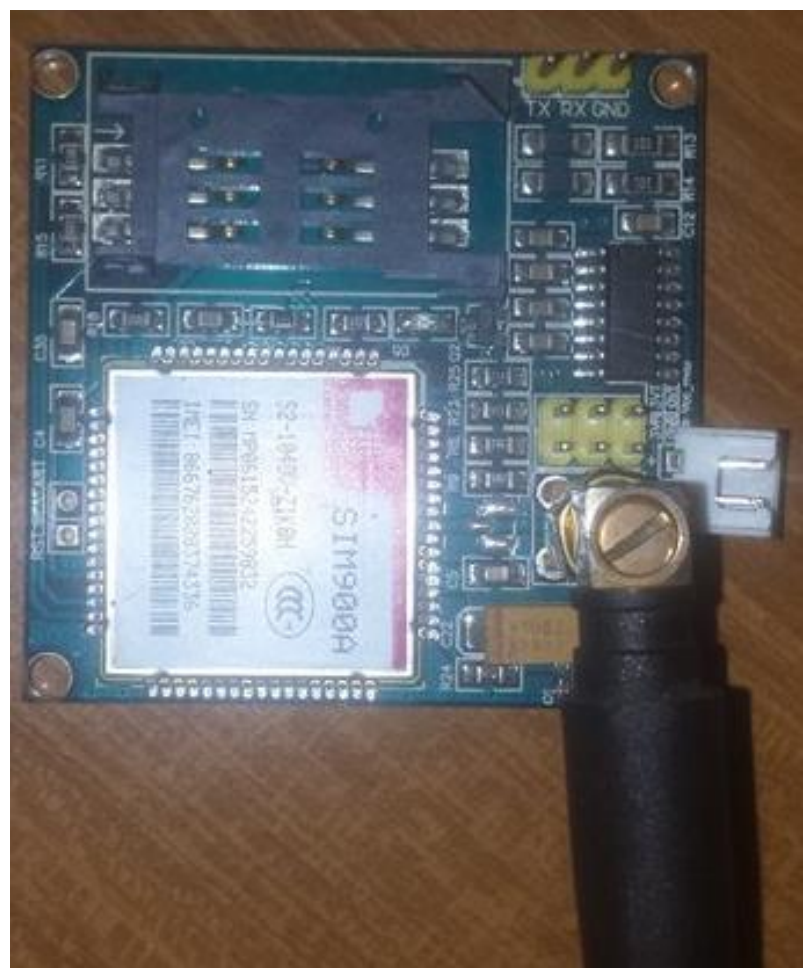

Fig. 4: GSM Sim900a by IC Station

The ADE-7755 is used to supply average power for the low frequency outputs. These logic output can be used to drive an electromechanical counter or MCU. The CF logic output giving real time and instantaneous average power making it applicable for calibration purpose or MCU interfacing. A power supply circuit is connected to the AVDD supply pin of $\mathrm{ADE} 7755$, and the energy metering IC remains in reset mode until the voltage at AVDD supply pin is not equal to greater to $4 \mathrm{~V}$; if the supply voltage falls below $4 \mathrm{~V}$, IC is reset and no pulse is issued to $F_{1}, F_{2}$ and $\mathrm{CF}$. The phase matching circuit ensures that there is a phase match between the channels of current and voltage regardless of the switching of HPF channel. In the absence of any load, the internal no threshold ensures that the output does not misbehave by giving garbage value. Fig. 5 shows the pin out of ADE7755.

\subsubsection{ACS-712(Current sensor)}

In order to achieve accurate and economical solution for current sensing for the project, the allegro current sensor module ACS-712 is used, which is commonly used in industrially, commercially and for telecommunication to monitor the current of the frameworks (Allegro, 2006). The main use of ACS-712 is to detect the switching of the generator, load management on the generator, overcurrent warnings and protection. The current module ACS-712 is an accurate, low-offset, linear hall sensor circuit having a copper conduction path, through which when current passed, a magnetic field 
is formed which is detected by the integrated Hall effect IC and converted into equivalent voltage. The reason for high accuracy of this sensor is the close proximity of the hall sensor (transducer) to the magnetic field. The accurate conversion of magnetic signal to voltage is performed by low offset chopper stabilized transducer (Allegro, 2006)

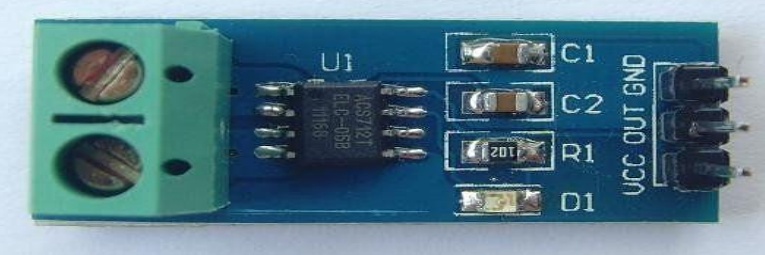

Fig. 5: ACS-712 module

DISPLAY UNIT: The display unit consists of a $16 \times 2$ LCD which is used to display the status of the open or closed lid along with the state of generator, load on the generator and fuel in the generator.

\subsubsection{Power module}

The power module is designed to power the complete system along with charging a battery and a cut off circuit to avoid overcharging of the battery. The power module initially provides 12VDC and 2.5 amperes to the system, which is regulated to 5VDC to power up the GSM module SIM 900a, using the same $5 \mathrm{VDC}$ pic $16 \mathrm{f} 877 \mathrm{a}$ is also powered, the $12 \mathrm{VDC}$ by the power module is used to power T/ll141 fuel sensor and charge the battery. The reason for providing 2.5 ampere current is that the GSM utilizes 2 amperes current during network detection or during receiving/sending of SMS.

\subsection{Working of project}

In this section, the complete working of the remote fuel monitoring system will be discussed briefly. As shown in the block diagram Fig. 1, the main component or controlling body of the flow of data is PIC-16f877a; a micro-switch is interfaced with the controller and acts a switch and is fitted at the lid of the generator. When the lid is closed, the switch is closed and is read as a closed circuit by the controller and a message of "LID IS CLOSED" is displayed and when the lid is opened the switch is also opened and the circuit is also opened and hence the controller gets no input (i.e. digital input) and hence a message "LID IS OPENED". The fuel sensor t/ll141 in connected to the analog port i.e. port A of the controller as the fuel sensor gives a voltage reading from $0-5$ VDC hence is directly interface able with the controller without any cutoff interfacing circuit, and is calibrated and a formula is derived which converts the input analog value into the fuel level of the tank (which varies for every tank). The load on the generator is determined by the ACS-712 module and the energy of the generator is determined by ADE-7755 as discussed in the previous section.
Now for the working of the system each and every scenario will be discussed below along with the process that takes place:

a) When the system is booted/ reset; System sends the state of the fuel lid (On or Off), State of the generator (ON or OFF) and the fuel level in the tank and it's price as shown in the Figs. 6 and 7.
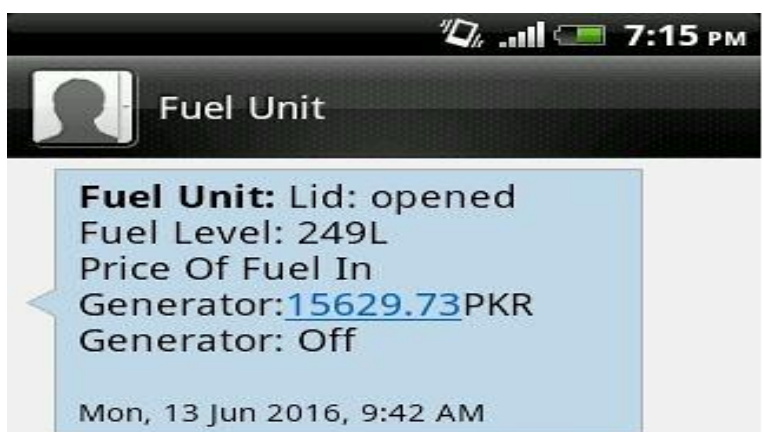

Fuel Unit: Lid: opened

Fuel Level: 249L

Price of Fuel In

Generator:15629.73PKR

Generator: Off

Mon, 13 Jun 2016, 9:42 AM

Fig. 6: Status of fuel lid: Open
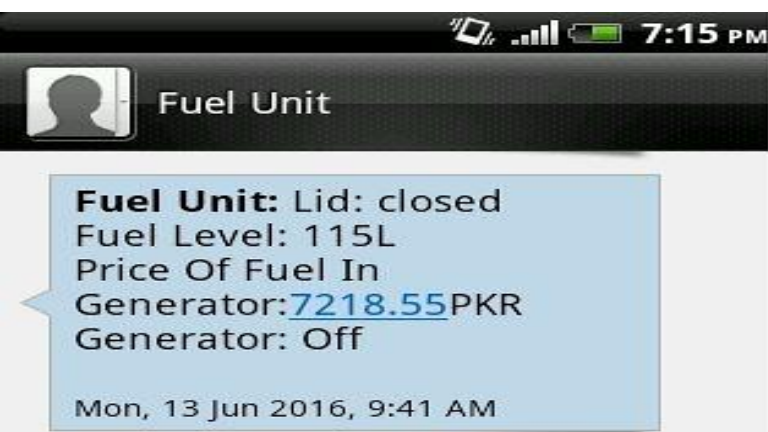

Fig. 7: Status of fuel lid: Closed

b) If the state of the button changes, i.e. if the lid was initially closed and is now opened, a message as seen in Fig. 8 is sent by the system and if the lid was previously opened and then closed then the message as seen in Fig. 9 is send by the system.

c) If the lid is opened and fuel level drops and the generator is not working, the message as seen in the Fig. 10 will be sent by the system.
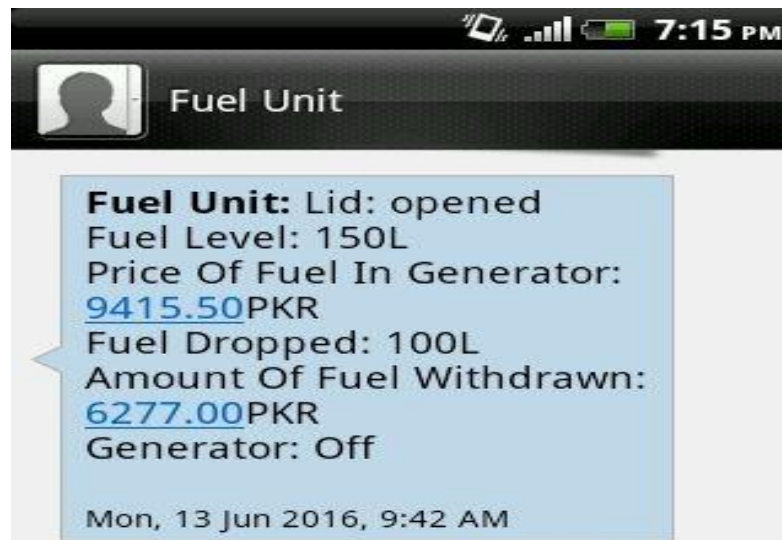

Fig. 8: Generator off and fuel lid open

d) If the lid is closed and the fuel level is dropped the message in Fig. 11 will be generated. 


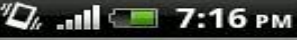

\section{Fuel Unit}

Fuel Unit: Lid: closed
Fuel Level: OL
Price Of Fuel In Generator:
O.0OPKR
Fuel Dropped: 34 L
Amount Of Fuel Withdrawn:
2134.18PKR
Generator: Off
Mon, 13 Jun $2016,9: 41$ AM

Fig. 9: Fuel lid closed and generator off

e) If the lid is opened and fuel level is increased i.e. the tank is refilled a complete summary will be send as seen the Fig. 12.
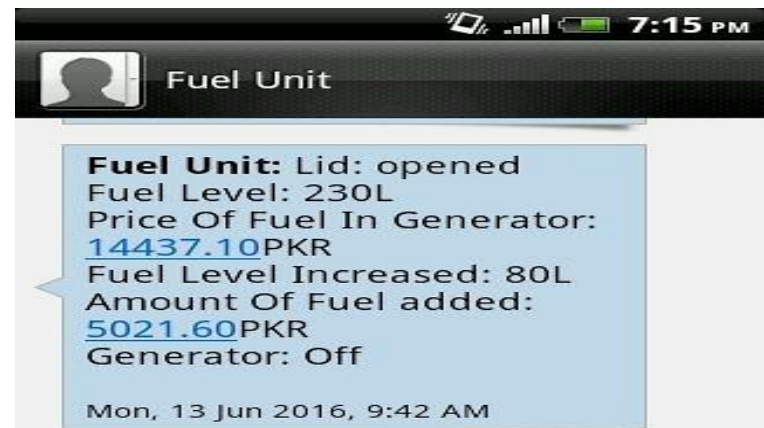

Fig. 10: Generator off and fuel lid open

f) If the generator is turned on the following message will be sent by the system as shown in the Figs. 13 and 14.

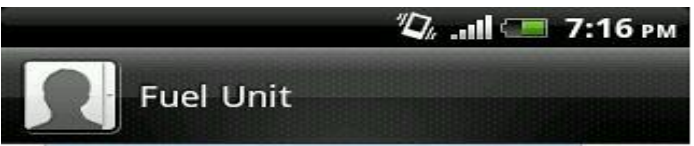

Fuel Unit: Lid: closed

Fuel Level: $127 \mathrm{~L}$

Price Of Fuel In Generator: 7971.79PKR

Generator: On

Total Load On The

Generator: 1.52

Sun, 12 Jun 2016, 9:57 PM

Fig. 11: Generator on and fuel lid closed

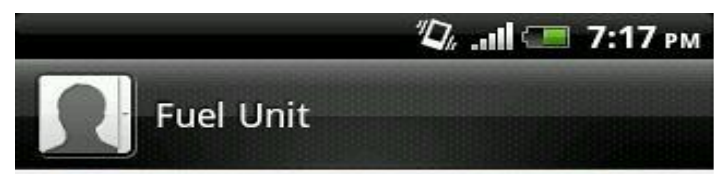

Fuel Unit: Lid: opened

Fuel Level: $250 \mathrm{~L}$

Price Of Fuel In Generator:

15692.50PKR

Generator: On

Total Load On The

Generator: 3.41

Sun, 12 Jun 2016, 9:56 PM

Fig. 12: Generator on and fuel lid open g) Now after the restoration of power the generator is turned off the following summary will be sent by the system as shown in Fig. 14 .
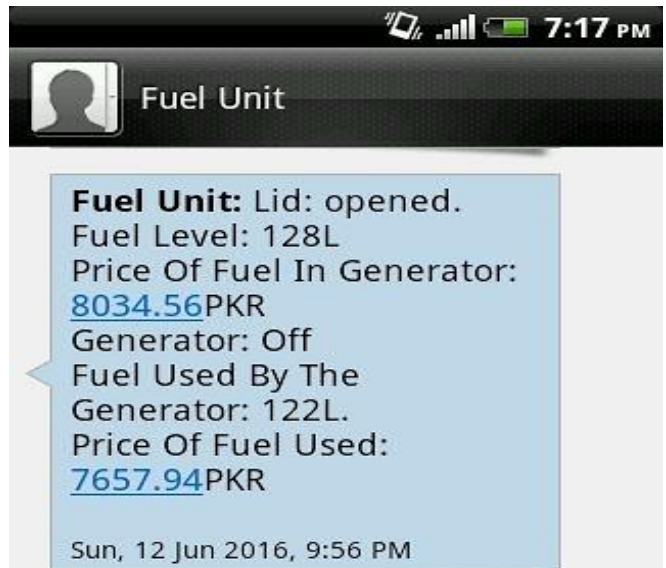

Fuel Unit: Lid: opened.

Fuel Level: $128 \mathrm{~L}$

Price Of Fuel In Generator:

8034.56PKR

Generator: Off

Fuel Used By The

Generator: $122 \mathrm{~L}$.

Price Of Fuel Used:

7657.94PKR

Sun, 12 Jun 2016, 9:56 PM

Fig. 13: Fuel Lid open and status of used fuel

h) The price of the fuel can be also adjusted remotely via SMS and is done by sending the fuel price in this form "ss <fuel_price>" and the following message will be generated by the system.

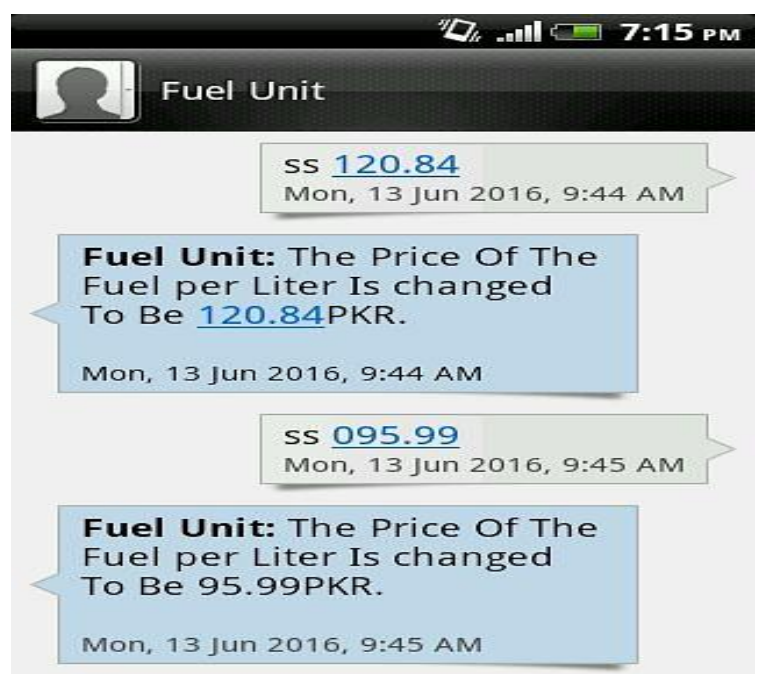

Fig. 14: Status of fuel

\section{Conclusion}

The proposed system is quite effective and unique in its features making it quite versatile and applicable in many applications such as banks and electricity generating companies, private and government corporations for monitoring and controlling the electrical generators being used, Ffurthermore, many features like automatic switching of the generator, temperature of the generator and a data logging system can also be incorporated and will be added in the next updated and advanced version of the system.

\section{Acknowledgment}

The authors would like to thank the Administration of NED University of Engineering \& 
Technology, Karachi Pakistan for providing resources to complete this research.

\section{References}

Aher SA and Kokate RD (2012). Fuel monitoring and vehicle tracking. International Journal of Engineering and Innovative Technology, 1(3): 166-169.

Allegro (2006). Fully integrated, hall effect-based linear current sensor IC with 2.1 Kvrms Isolation and a low-resistance current conductor (ACS712). Available online at: www.allegromicro.com/ /media/Files/Datasheets/ACS712Datasheet.ashx

Biao MA (2007). Design of electricity power measurement and management system for student apartment based on Ade7755 [J]. Computer Engineering, 2: 072. Available online at: http://en.cnki.com.cn/Article_en/CJFDTOTAL-JSJC20070207 2.htm
Garg VK and Rappaport TS (2001). Wireless network evolution: $2 \mathrm{~g}$ to $3 g$. Prentice Hall, Upper Saddle River, USA.

Harijan K, Uqaili MA, and Memon M (2008). Renewable energy for managing energy crisis in Pakistan. In The International Multi Topic Conference, Springer Berlin Heidelberg, Heidelberg, Germany: 449-455.

Iovine J (2004). Pic microcontroller project book. McGraw-Hill, New York, USA.

Predko M (1997). Programming and customizing the PC microcontroller. McGraw-Hill, New York, USA.

Zaghloul MS (2014). GSM-GPRS arduino shield (Gs-001) with SIM 900 chip module In Wireless data transmission system for data acquisition and control of power induction furnace. International Journal of Scientific and Engineering Research, 5(4): 776-780

Zvonar Z, Jung P, and Kammerlander K (1998). Gsm: Evolution towards 3rd generation systems. Springer Science \& Business Media, Berlin, Germany. 